\title{
Silicon High-Speed Electro-Optic Modulator
}

\author{
L. Alloatti, D. Korn, D. Hillerkuss, T. Vallaitis, J. Li, \\ R. Bonk, R. Palmer, T. Schellinger, C. Koos, \\ W. Freude, J. Leuthold \\ Institute of Photonics and Quantum Electronics (IPQ), \\ Karlsruhe Institute of Technology (KIT), \\ 76131 Karlsruhe, Germany, \\ luca.alloatti@kit.edu
}

\author{
A. Barklund, R. Dinu, J. Wieland \\ GigOptix Inc. \\ Switzerland and Bothell (WA), USA
}

\author{
M. Fournier, J. Fedeli \\ CEA, LETI \\ 38054 Grenoble, France
}

\author{
W. Bogaerts, P. Dumon, R. Baets \\ Photonics Research Group, Ghent University \\ IMEC, Dept. of Information Technology, \\ B-9000 Gent, Belgium
}

\begin{abstract}
A $40 \mathrm{Gbit} / \mathrm{s}$ electro-optic modulator is demonstrated. The modulator is based on a slotted silicon waveguide filled with a nonlinear organic material. A modulation voltage-length product of $V_{\pi} L=0.21 \mathrm{Vcm}$ can be achieved.
\end{abstract}

Keywords - electro-optic; modulator; silicon; CMOS; slot, socket, high-speed, chromophores, second order nonlinearity

\section{INTRODUCTION}

Silicon-on-insulator (SOI) photonics is considered a promising option for lowest-cost highly integrated photonic circuits. Actual research is oriented towards complementing the CMOScompatible optical toolbox. A fast, inexpensive modulator with low RF power requirements is one of the key components that needs more attention. So far, silicon photonics high-speed modulators exploit the plasma effect $[1,2,3]$, because silicon lacks $\chi^{(2)}$-nonlinearity. However fundamental speed limitations related to carrier injection and removal are likely to become limiting factors in this technique.

To avoid these limitations, a silicon-organic hybrid (SOH) approach has been suggested. It combines silicon waveguides with a highly nonlinear electro-optic (EO) organic material [4-9]. This technology allows to choose among a variety of materials with significantly larger $\chi^{(2)}$-susceptibility than even $\mathrm{LiNbO}_{3}[10-12]$.

This work presents the first successful implementation of a silicon EO modulator with $40 \mathrm{Gbit} / \mathrm{s}$ modulation speed. The modulator potentially operates at low voltages due to the slotand-socket structure (Fig. 1). It features a strong optical field enhancement inside the low-index slot that is filled with the nonlinear material. In addition, the modulation is applied via conductive sockets and therefore drops entirely across the slot. The modulator operates over the entire C-band in contrast to resonant devices.

\section{DESIGN AND FABRICATION}

The structure of the modulator is shown in Fig.1. It consists of a slot waveguide and two $70 \mathrm{~nm}$ thick doped silicon slabs (sockets), which electrically connect the optical waveguide to the metal electrodes. The RF voltage drops essentially across the $120 \mathrm{~nm}$ wide slot. Simulations indicate that the free-carrier induced optical loss can be kept below $1 \mathrm{~dB} / \mathrm{mm}$ while preserving an electrical bandwidth larger than $100 \mathrm{GHz}$. By using a nonlinear material with $r_{33}=150 \mathrm{pm} / \mathrm{V}$ a voltage-length product of $V_{\pi} L=0.21 \mathrm{Vcm}$ can be achieved. This value is seven times smaller than that published most recently for a $12.5 \mathrm{Gbit} / \mathrm{s}$ plasma effect modulator [3].

The modulator has an overall length of $2.7 \mathrm{~mm}$. The phase modulator section is $1.7 \mathrm{~mm}$ long. Two rib-to-slot converters,

(a)

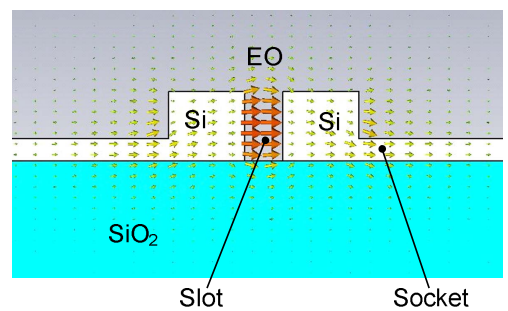

(b)

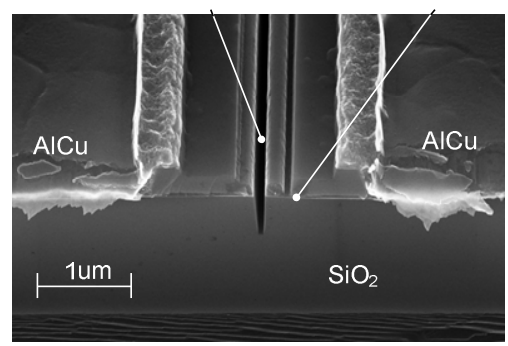

Figure 1. Structure of phase modulator (a) Optical electric field for the quasi-TE mode of the socket waveguide. The field is confined mainly to the slot. Because of the conductive sockets the RF voltage drops mainly across the slot where the nonlinear material is located and the optical field strength is large. (b) SEM picture of the cross section of a socket waveguide. 


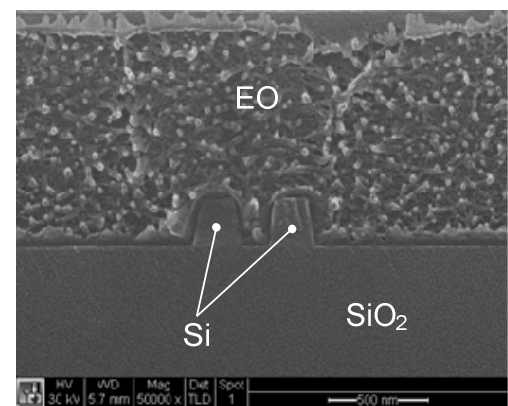

Figure 2. SEM image of a slot waveguide filled with the electro-optic material. The slot is completely filled.

two tapers and two grating couplers connect the phase modulator to external fibers. The wafer has been fabricated within the ePIXfab framework by CEA-LETI according to standard processes of the microelectronic industry. The silicon has been implanted with two different doses: A low dose for all optical regions (including the grating couplers), and a high dose for ensuring ohmic contact between the metal electrodes and the silicon sockets. The $\mathrm{AlCu}$ electrodes have been structured by means of an oxide hard mask, which had to be opened after metallization for filling the slot with the organic material. This process, which we are currently optimizing, increased the fiberto-fiber loss from $23 \mathrm{~dB}$ to $39 \mathrm{~dB}(2 \times 5 \mathrm{~dB}$ of which are due to the grating couplers). The doping of all the optical regions explains the $23 \mathrm{~dB}$ insertion loss. We believe that by doping the phase shifter only and by improving the grating couplers a fiber to fiber loss below $10 \mathrm{~dB}$ is achievable.

The waveguides have been coated (Fig. 2) with an electrooptic organic material having a nominal electro-optic coefficient of $r_{33}=150 \mathrm{pm} / \mathrm{V}$ [10]. The same material is currently used in the production line of GigOptix. The EO polymer has been poled at its glass-transition temperature by applying $12 \mathrm{~V}$ at the same electrodes used for modulation. The data presented in this paper correspond to an electro-optic coefficient of $r_{33}=3 \mathrm{pm} / \mathrm{V}$. In a similar device, but without RF electrodes, we achieved a nonlinearity of $r_{33}=14 \mathrm{pm} / \mathrm{V}$. We are currently working on improving the poling efficiency of the electrooptic material.

\section{EXPERIMENTAL CHARACTERIZATION}

\section{A. Frequency response}

For measuring the frequency response of the phase modulator we applied a sinusoidal voltage with frequencies between $15 \mathrm{GHz}$ and $40 \mathrm{GHz}$. The chip was contacted with a groundsignal RF probe. The RF power at the probe input was kept constant to $10 \mathrm{dBm}$. The resulting phase modulation index $\eta$ was determined by evaluating the power ratio between optical carrier and first optical sideband. The results are shown in Fig. 3. The variation of the modulation index is smaller than $13 \%$ over the entire modulation frequency range. This demonstrates the high-speed capability of our device. The modulator is also optically broadband: Fig.4 demonstrates a constant modulation index over the entire C-band.

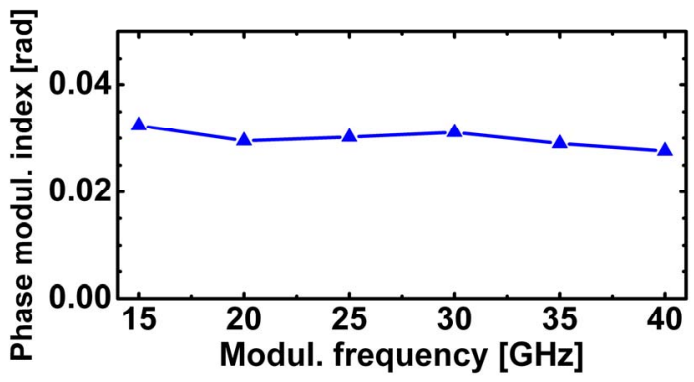

Figure 3. Phase modulation index $\eta$ as a function of the modulation frequency for a constant RF amplitude of $1 \mathrm{~V}$. The phase modulation index varies less than $13 \%$ showing high-speed capability.

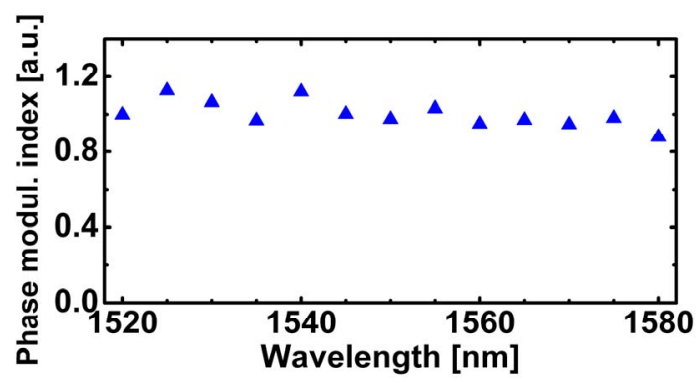

Figure 4. Phase modulation index $\eta$ as a function of wavelength. The phase modulation index is constant over more than the entire C-band. The modulation frequency $(20 \mathrm{GHz})$ and amplitude are fixed.

\section{B. Modulation with PRBS data}

For demonstrating the capabilities of the phase modulator, it was driven with a $42.7 \mathrm{Gbit} / \mathrm{s}$ data signal (PRBS length $2^{7}-1$ ). The device consists of the on-chip phase modulator, and an external delay-interferometer (DI) for converting phase modulation to amplitude modulation (Fig. 5). The measured signal quality is $\mathrm{Q}^{2}=12.7 \mathrm{~dB}$. A BER $=2 \times 10^{-6}$ is measured using a $70 \mathrm{GHz}$ photodiode and a $42.7 \mathrm{Gbit} / \mathrm{s}$ bit error ratio tester (BERT). This is well below the FEC threshold of $2 \times 10^{-3}$.
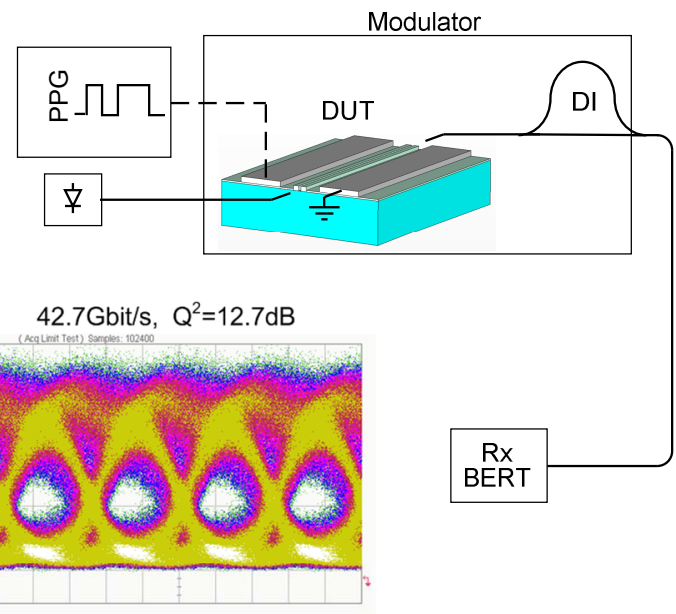

Figure 5. Characterization setup. $42.7 \mathrm{Gbit} / \mathrm{s}$ data is generated by a pulse pattern generator (PPG). The phase modulation produced by the DUT is converted to amplitude modulation by means of an external delay interferometer (DI).The signal is then detected using a pre-amplified receiver. $\mathrm{A} \mathrm{BER}=2 \times 10^{-6}$ has been achieved. 


\section{ACKNOWLEDGMENTS}

We acknowledge support by the DFG Center for Functional Nanostructures (CFN), the KIT Initiative of Excellence, the Karlsruhe School of Optics and Photonics (KSOP), the EUFP7 projects EURO-FOS (grant 224402) and SOFI (grant 248609), and by the BMBF joint project MISTRAL, funded by the German Ministry of Education and Research under grant 01BL0804. We are grateful for technological support by the Light Technology Institute (KIT-LTI) and the ePIXfab (silicon photonics platform). We thank J. Groß and S. Schneider (both KIT-IPQ) for helping with the modulator design.

\section{REFERENCES}

[1] L. Liao et al., Electron. Lett., vol. 43, no. 22, 20072253, 2007. doi:10.1049/el:20072253

[2] W. M. J. Green et al., Opt. Express, vol. 15, no. 25, 17106-17113, 2007. doi:10.1364/OE.15.017106

[3] N. N. Feng et al., Opt. Express, vol. 18, no. 8, 7994-7999, 2008 doi:10.1364/OE.18.007994

[4] M. Hochberg et al., Opt. Express, vol. 15, no. 13, pp. 8401ff, June 2007. doi:0.1364/OE. 15.008401

[5] C. Koos et al., ECOC 2007, paper P056.

[6] J.-M. Brosi et al., Opt. Express, vol. 16, no. 6, 4177-4191, 2008. doi:10.1364/OE.16.004177

[7] T. Baehr-Jones et al., Appl. Phys. Lett., vol.92, 163303, 2008. doi:10.1063/1.2909656

[8] J. Leuthold et al., Proc. of the IEEE, vol. 97, no. 7, 1304-1316, 2009. doi:10.1109/JPROC.2009.2016849

[9] J.H. Wülbern et al., APL, vol. 94, 241107, June 2009. doi:10.1063/1.3156033

[10] E. M. McKenna et al., J. Opt. Soc. Am. B, vol. 24, no. 11, 2888-2892, 2007. doi:10.1364/JOSAB.24.002888

[11] H. Chen et al., Appl. Phys. Lett., vol. 93, 043507, 2008. doi:10.1063/1.2965809

[12] B.-J. Seo et. al., J. of Lightw. Technol., vol. 27, no.15, pp. 3092-3106, Aug. 2009. doi:10.1109/JLT.2008.2005916 